\title{
FROM CONCEPT DEVELOPMENT TO IMPLEMENTATION: CHOOSING BY ADVANTAGES ACROSS AN ORGANIZATION
}

\author{
Annett Schöttle ${ }^{1}$, Luisa Maria Gigler ${ }^{2}$, and Brent Mingle ${ }^{3}$
}

\begin{abstract}
Decision-making is one of the most important and underestimated activities of every organization. Decisions define the commitment of our most limited resources and are the determining factor between success and failure. How individual decisions are taken directly impacts the decision outcome. Therefore, the method used to decide is of utmost importance. Choosing by Advantages (CBA) is a decision-making method that has been growing in application and acceptance in the last few years within the International Group for Lean Construction (IGLC) community. Existing publications regarding CBA mainly discuss the benefits of the method in theory and in practice, but none of them explain how the method was applied nor if it was also broadly implemented across organizations to the different project levels. This paper closes the gap by giving guidance and insights on the development and rollout of CBA trainings for the construction industry based on the case study of Daimler AG. The authors conclude that the implementation of CBA requires a thoughtful plan that leaves room for adjustments in accordance to the project team's needs.
\end{abstract}

\section{KEYWORDS}

Change, Choosing by Advantages, Culture, Decision-making, Design Thinking.

\section{INTRODUCTION}

Baron $(2008$, p. 6) defines a decision as a "choice of action - of what to do or not to do. [...] made to achieve goals, [...] based on beliefs about what actions will achieve the goals". Decision-making can be defined as "irrevocable allocation of resources, irrevocable in the sense that it is impossible or extremely costly to change back to the situation that existed before" (Howard 1966, p. 97). Both quotes show how crucial decisions and the decisionmaking process are for every organization, because decisions guide the direction and give orientation. Thus, the question of how to make decisions across an organization that fit the purpose and are understandable to all affected parties arises. Suhr (1999) focuses on how

1 Senior Consultant, Refine Projects AG, Schelmenwasenstraße 34, 70567 Stuttgart, Germany, +4915156561529, annett.schoettle@ refineprojects.com

2 Process Analyst, Refine Projects AG, Stuttgart, Germany, luisa.gigler@ refineprojects.com

3 Pacemaker Decision Making, Leadership 2020, Daimler AG, 70546 Stuttgart, Germany, +4917630971873 brent.mingle@ daimler.com 
to make decisions and states that "[o]ur methods produce our decisions. Our decisions guide our actions. And our actions cause our outcomes" (p. 17). Therefore, to tangibly improve the outcome of decisions, decision-makers have to understand why and how decisions are made. Not knowing the reason for decisions can easily result in a lack of commitment, inaccuracy, or even sabotage against decisions during the implementation phase. Elmuti et al. (1993) emphasize that meaningless or thoughtless decisions are an indicator for, but also cause of, poor communication, invisible leadership and thus a lack of teamwork. Many collaborative decision-making committees suffer when trying to explain the why behind decisions, which leads to frustration and a loss of trust within the collaborative decision-making culture. Employees and leaders alike often report a high level of frustration regarding decision-making. At many large organizations, like Daimler $\mathrm{AG}$, the multiple layers of required bureaucratic alignments lead to an ambiguous and vague understanding of the decision premise and logic which then leads to decisions not being taken, resulting in an unclear decision purpose with a low level of commitment. Therefore, it is important to install a transparent and traceable decision-making process which helps team members to participate in the decision-making or at least understand the reasons and logic behind decisions. Moreover, confident decision-making by a collaborative team will automatically result in a team that is willing to take ownership and who will carry the consequence of decisions taken.

Decisions and decision-making are key not only for industries such as the automotive industry, but also for proceedings in construction projects. Therefore, based on four cases where two projects practically applied CBA to decide between alternatives, Schöttle et al (2018) explain how the decision-making process should be designed to be effective in construction projects. As part of the process, the authors recommend applying CBA as the decision-making method. In addition to this paper, many other papers have demonstrated the benefits regarding CBA in comparison to other decision-making methods theoretically (e.g. Schöttle and Arroyo 2017). All the papers have in common is that they demonstrate the benefits of applying CBA either theoretically or based on a single case. None focus on a strategic widespread implementation across an organization or the construction industry as a whole, although the construction industry is not using its potential to implement new methods and tools quickly through project teams. Therefore, this paper focuses on how to develop a training concept for CBA and how to rollout the method organization-wide. Thus, after a short explanation of CBA and design thinking as well as the research method, the strategic implementation of CBA based on an individual developed training concept for Daimler AG is described. Challenges, findings and learnings from the case are then discussed and theoretically transferred to the construction industry to support the CBA implementation and give guidance for a successful implementation in construction. Finally, the conclusion will be drawn in reference to the research questions.

\section{BACKGROUND LITERATURE REVIEW}

\section{Choosing By Advantages}

CBA is a multi-criteria decision-making system developed by Shur (1999) that is based on a clear defined vocabulary and an anchored judgement which compares the advantages of 
alternatives. Independent of the decision complexity, the CBA system provides different methods. Two methods are the: (1) Two-list method and the (2) Tabular method. The CBA Two-list method consist of five steps (see Figure 1) and is applicable for decisions with less complexity.

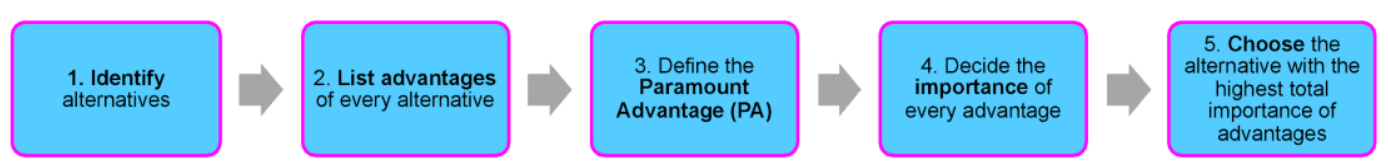

Figure 1: Steps of CBA Two-list Method

If decisions are more complex, meaning that a decision consists of various information, data and alternatives, requires clear documentation, and involves different participants, the CBA Tabular method should be applied (see Figure 2), because it helps to understand the different perspective of the team members. It was reported that using the Tabular method leads to productive conversation, because of less tangential discussions (Arroyo and Long 2018), results in constructive debates, less misinterpretation, and a transparent documentation that is traceable for a party not involved in the decision-making (Schöttle and Arroyo 2017). Furthermore, teams achieved faster consensus and felt less frustration during decision-making when using CBA in comparison to scoring systems (Arroyo et al. 2016) and felt more confident about the decision quality (Schöttle et al. 2018).

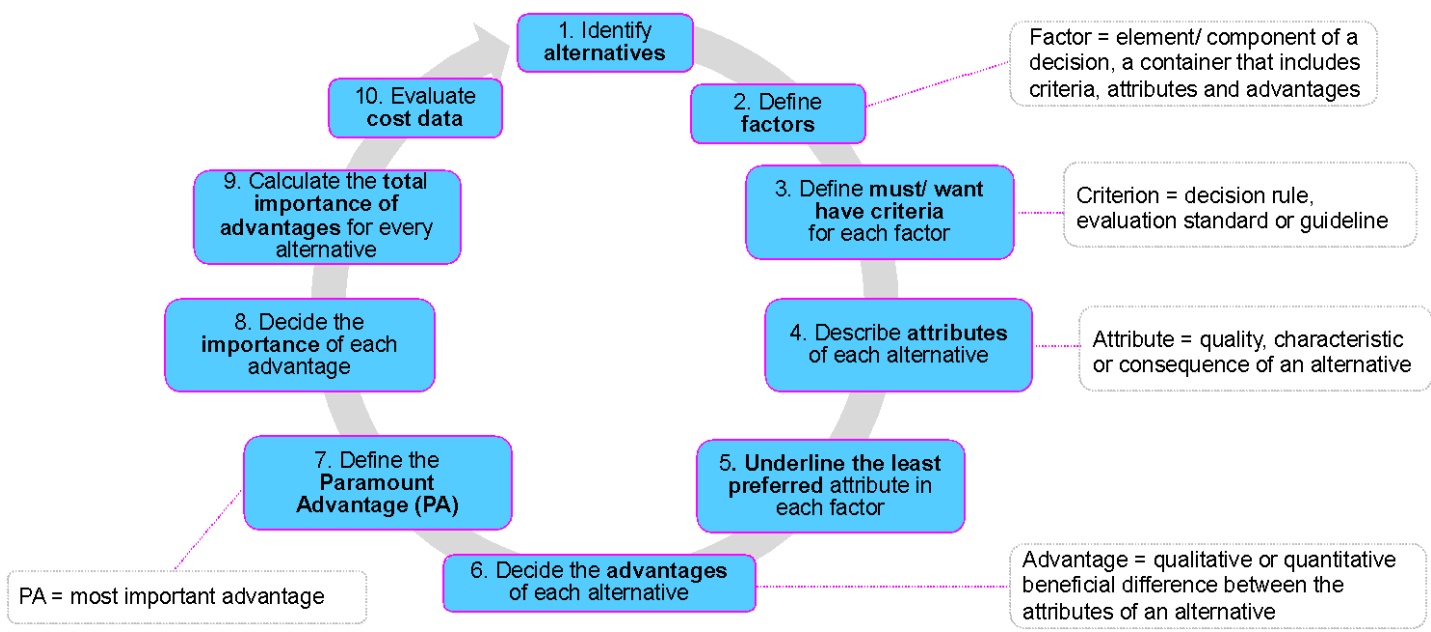

Figure 2: Steps of CBA Tabular Method (based on Arroyo 2014) with vocabulary

\section{DESIGN THINKING}

Using the attitude and methods from art and design, Design Thinking (DT) is an incremental and iterative approach to identify human needs and develop new and unexpected ideas based on rational and end-user-focused problem solving (Kelley and Kelley 2013; Brown 2008). Through the application of various creativity techniques and rapid conceptual prototyping, DT describes an effective toolkit when looking for new opportunities or overcoming complex challenges (Meinel and Leifer 2011). Referring to the DT Model of the Hasso-Plattner-Institute, the DT process consists of two phases with 
six steps (see Figure 3). The main idea of the orientation phase is to gather as much information as possible about the problem and potential user groups as well as to structure and synthesize them into a few but central insights. Based on these insights, concrete ideas are developed in the second, solution-oriented, phase.

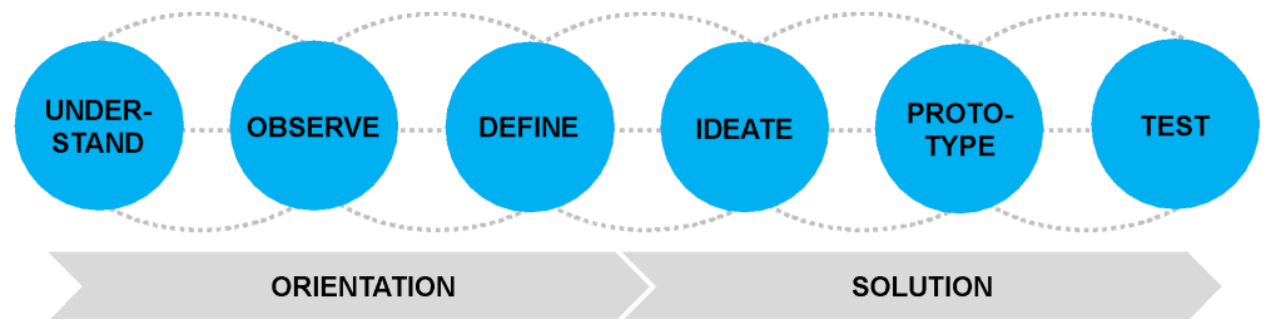

Figure 3: The Design Thinking Model (based on Plattner et al. 2009)

\section{RESEARCH QUESTIONS AND METHOD}

The purpose of this research has two objectives. First, understanding the requirements and the user needs to develop a concept for an organization-wide implementation of CBA at Daimler AG. Second, to transfer the findings to the construction industry. Therefore, action research is used to investigate issues and act based on the findings (Dickens and Watkins 1999). The authors of this paper also developed the training concept for Daimler AG. To solve issues which occurred during the development phase and to improve the concept, the authors conducted data through Plus Delta, reflection, short surveys, discussions and direct feedback with attendees based on three main questions: (1) How to implement CBA effectively? (2) How to train users and trainers in the CBA method effectively? (3) How to globally rollout CBA across a whole organization? These three questions will be answered by describing the developed concept and the current status of implementation at Daimler AG. To transfer the learnings and findings to the construction industry, the authors use one research question, which includes two sub-questions: (4) How can the training concept be transferred to the construction industry? (4a) Are there challenges that need to be considered in comparison to the automotive industry? (4b) Does the concept need to be adjusted?

\section{CASE STUDY: CBA IMPLEMENTATION AT DAIMLER AG}

Daimler AG is a German multinational automotive company with a headquarter based in Stuttgart, Germany. Due to fast-changing market developments in the mobility provider industry, in 2015, Daimler AG launched the bottom-up initiative Leadership 2020 to modernize and transform its aging leadership culture and cumbersome organizational interactions while achieving cultural resilience and flexibility for the future. The initiative identified Decision Making as one of the eight Game Changers necessary to tangibly realize empowerment, agility and trust within the leadership culture. The Game Changer Decision Making promotes empowering competency within decisions as opposed to the classical cascading hierarchal process. Over 50 global decision processes have been optimized to empower decision-making at the lowest level possible while making them traceable, trustworthy and sound. To achieve this, appropriate tools and methods are 
necessary to: (1) make better decisions in a collaborative team and (2) to make better decision proposals. Figure 4 presents an overview of the CBA implementation. First, a half-day training workshop was developed; second the rollout concept was created to give more insights regarding CBA and help users with the implementation; and, third, a train the trainer (TTT) concept was established to multiply the implementation across the organization.

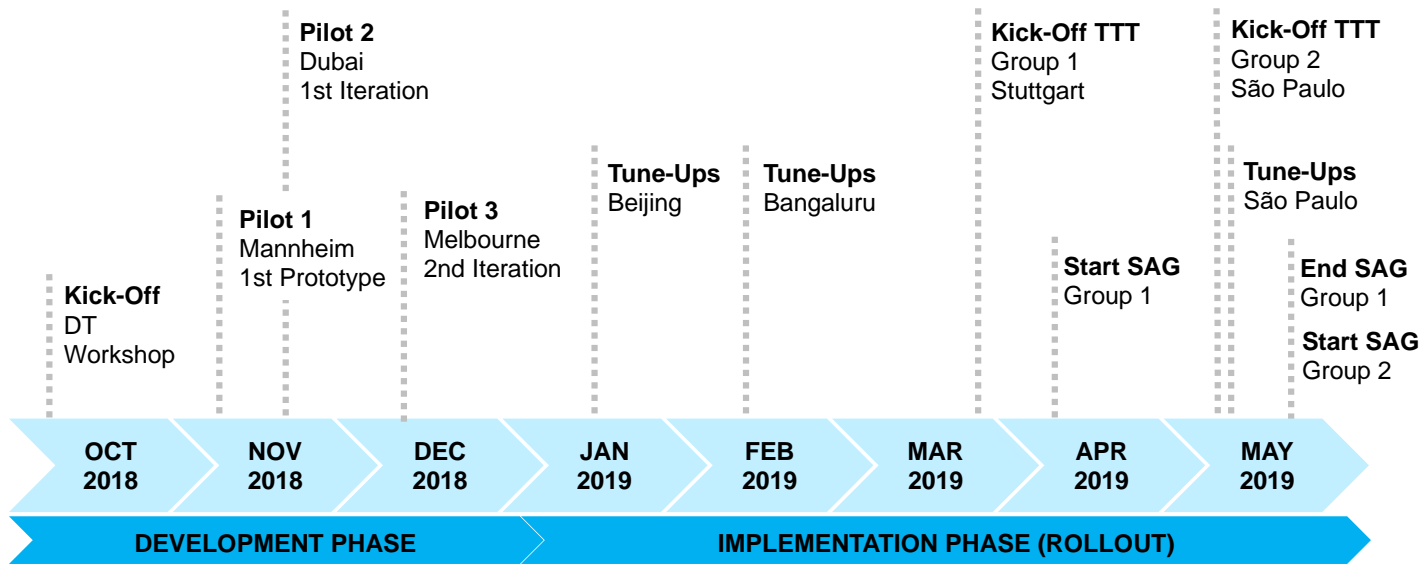

Figure 4: Overview of the concept development and implementation

\section{DeVelopment Phase}

Considering the deficient experiences with CBA implementation across an organization, the authors defined an iterative process to develop the concept. Two main elements of the approach are an initial DT workshop and the evaluation of various pilot tests with PlanDo-Check-Act (PDCA) cycles. Results and recommendations from both elements served as orientation framework for the first concept draft as well as for the rollout and adjustment of the CBA training concept within the implementation phase. Thus, the development phase started with a DT workshop to identify the individual needs and demands of the users concerning their decision-making pain points and their learning preferences. Five team members of the global Decision Making squad (including the third author) participated in the Stuttgart workshop, which was facilitated by the first and second authors. In order to physically visualize work in progress and results of the workshop, the team used the approach of a design wall which facilitates every step of the DT process (see Figure 5). First, potential user groups of different management levels were defined. Second, mindsets of the user groups were emphasized to define their specific point of view. Third, with the user point of view as reference, the ideation process followed to develop feasible ideas for a training concept as well as suitable training approaches and tools. During the workshop, important constraints and needs for the training (e.g. time, pre-load examples, or the need for a follow-up after a training workshop) were identified.

Considering the insights of the DT workshop, the first prototype of a four-hour CBA workshop (so called Tune-up) was developed. As DT is described as an iterative process itself, several iteration steps are necessary during the development phase in order to adjust and improve the developed prototype. Within two months, a total of six four-hour 
workshops were held in different international branches of Daimler (see Figure 4). Using PDCA cycles, the CBA team was able to collect deliberate feedback on specific aspects of the training and to continuously improve the workshop based on the learnings (see Table 1). Both the DT workshop and the PDCA ensured the iterative development of the training, closely linked to the individual organizational demands and needs. After major improvements, the workshop was carried out three times in Beijing, four times in Bengaluru, once in Germersheim, and twice in São Paulo, at various management levels and departments (R\&D, IT, Production, etc.).

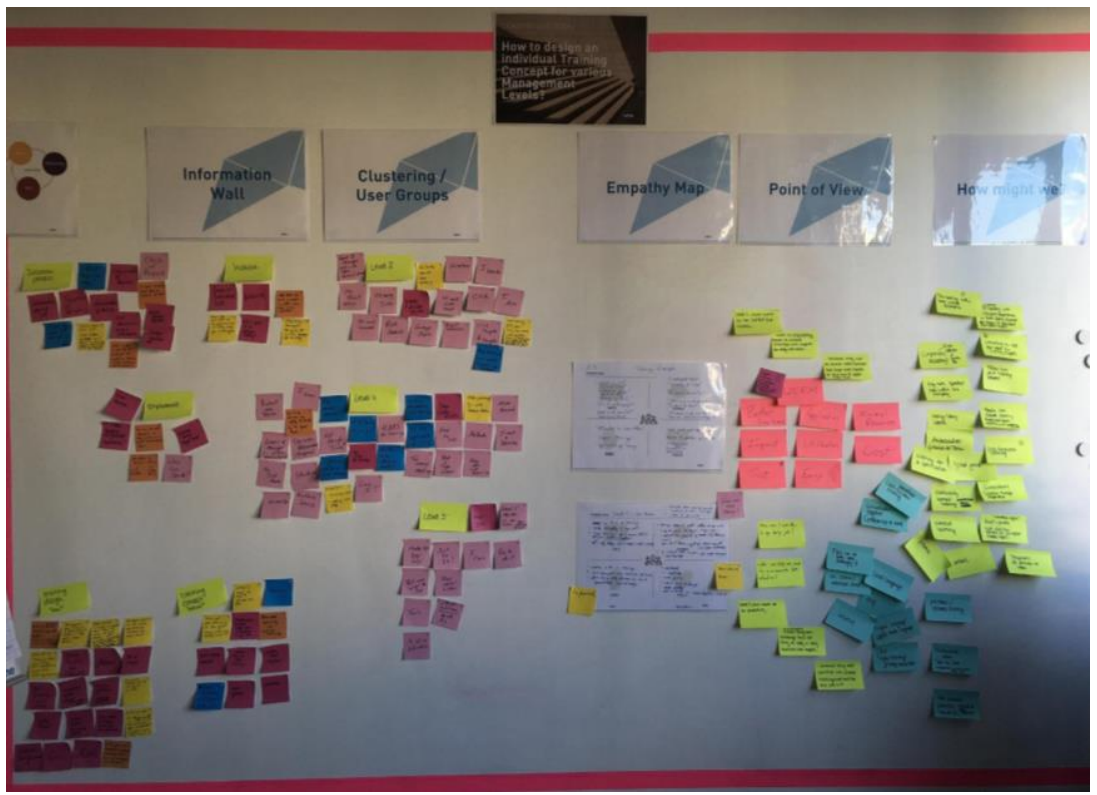

Figure 5: Design wall as result of the Design Thinking workshop

Table 1: PDCA during development phase

\begin{tabular}{|c|c|c|}
\hline Pilots & \# Tune-ups & Major learnings \\
\hline Mannheim & 1 & $\begin{array}{l}\text { - } \quad \text { More visualization and active Two-list exercise } \\
\text { - } \text { Short explanation of terms and an example for pre-load } \\
\text { - Present the difference to weighting rating calculating (WRC) } \\
\text { - } 2 \text { pages with the essentials of CBA as follow-up } \\
\text { - } \text { Frame the topic in context to Leadership } 2020 \text { and the } \\
\text { Decision Making squad }\end{array}$ \\
\hline Dubai & 1 & $\begin{array}{l}\text { - Better explanation of sound decision-making } \\
\text { - } \text { Figure with the steps of the Tabular Method next to the } \\
\text { template for the Tabular exercise } \\
\text { - Simplify the theoretical part of the presentation }\end{array}$ \\
\hline Melbourne & $\begin{array}{c}4 \\
\text { (in } 2 \text { days) }\end{array}$ & $\begin{array}{l}\text { - Max. number of workshop participants } \\
\text { - Max. number of group participants for CBA Tabular exercise } \\
\text { - More visualization of the examples in the presentation }\end{array}$ \\
\hline
\end{tabular}




\section{IMPLEMENTATION PHASE: ROLLOUT}

Continuous iterative cycles based on the evaluation within the framework of PDCA identified that the rollout of the CBA workshops across the organization had to be customized by consulting with the coordinator and management sponsor for the department. Therefore, the major learnings from the various pilots continuously improved and enhanced the initial prototype of the four-hour training workshop. As one of the most important learnings, additional pre-load and follow-up elements were required and have been integrated into the concept. The pre-load prepares the users with a brief pre-reading and a simple exercise to gain their first experience with CBA and to develop real decision examples that are used to exercise the Tabular method in order to make the training more comprehensible, tangible and work-related. Afterwards, the follow-up provides the users with essential knowledge, further reading and support options. Figure 6 summarizes the steps and contents in detail. The training concept helped the users to understand the need for a sound decision-making method and prepared them to apply CBA correctly. Furthermore, participants got motivated and enthusiastic about using CBA in daily business.

The rollout phase started in January 2019 with three workshops with different management levels and departments in Beijing. Especially, the third workshop was further customized to meet the needs of the newly developed team to reflect on their decisionmaking culture and to improve their collaborative decision process. In comparison to the other two workshops, this workshop was primarily composed of local Chinese participants from the mid-lower management levels. It was important to give them the room to articulate their pain points and their frustrations regarding decision-making using the nominal group technique. Their local decision-making cultural pain points revolved around difficulties in communicate specific market issues with the upper management levels that usually consist of German headquarter-centric perspectives. This underscores that, in both the development and the implementation phase, iterative processes appear to be crucial in order to secure and enhance the success and required adaptability of the CBA training concept.

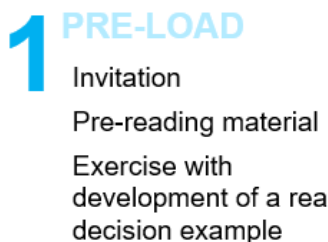

decision example

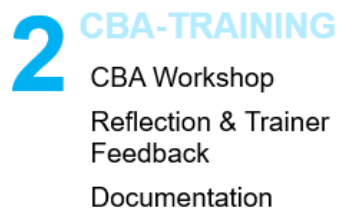

Reflection \& Traine

Documentation

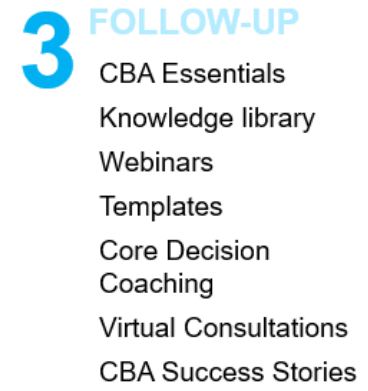

CBA Success Stories

Figure 6: Training approach to train the users

\section{IMPLEMENTATION PHASE: Train The Trainer}

To foster internal interested parties to become CBA trainers themselves, an additional training concept was developed. The concept includes capability training and coaching for 
CBA as well as facilitator skills. As shown in Figure 7, the approach is composed of three units: (1) knowledge, (2) partner coaching, and (3) advice and support. Unit one prepares the essential concept knowledge through a Kick-off and a Study Action Group (SAG). During the Kick-off and the SAG the participants have to perform exercises to build knowledge and prepare for the facilitation. Moreover, the first unit helps to develop a supportive network between the trainers that creates unit three. Furthermore, trainers get help in preparation decisions such as ready-use templates. Unit two supports the participant to become a trainer ideally within the framework of a three-step-flow partner coaching. Unit three offers additional support and enables qualified trainers to facilitate decisions with CBA. By completing all four pink steps successfully, the participant receives a CBA trainer certificate.

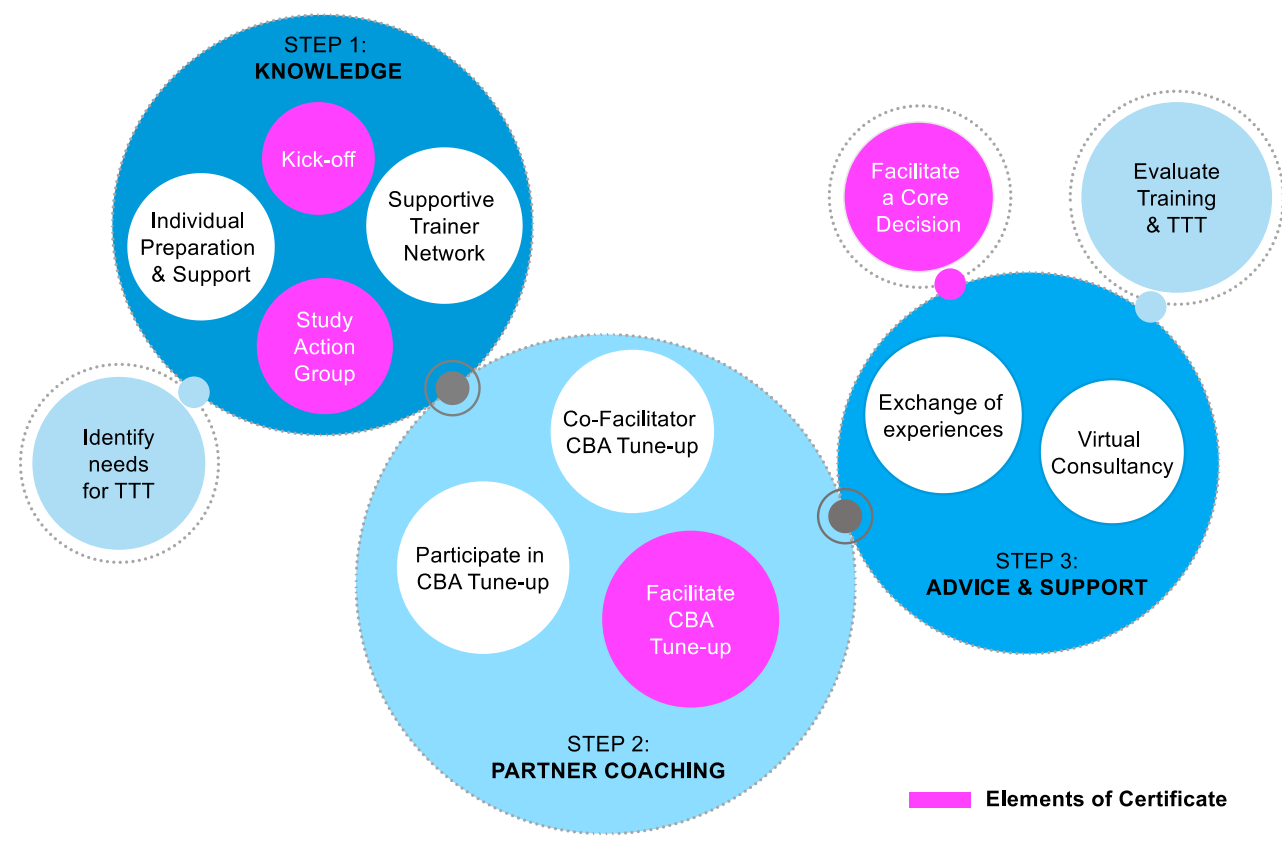

Figure 7: Training approach to train the trainer

\section{FINDINGS AND DISCUSSION}

The approach of the Daimler case study provides a broad expert network within Daimler with internal specialists and contact partners for any kinds of questions and concerns about CBA with a strong relevance for the local decision-making culture. The network enables a continuous exchange of knowledge and experiences and is therefore the basis for multiplication. Moreover, the intense evaluation of the feedback enabled continuous improvement, making it possible to deliver a customized and adaptive concept ready to be implemented across the organization. It can be seen that considering cultural difference is important to successfully train CBA in a global work environment. Furthermore, it is also important that potential users can identify and relate to how the CBA method can help them individually, while recognizing its operational benefits. CBA is introduced in a hands-on, content rich and time-efficient workshop format where the participants exercise the two- 
list method and the Tabular method. Within the workshop the group's decisions were presented by a member of an adjacent decision-example group so that the reliable transparency and the traceable documentation of the CBA method decisions could be experienced. Workshop participants consistently stated that they were able to understand why a group decided how they decided based on the Tabular method of documentation and the easy-to-perceive differentiation between the alternatives. Moreover, during decisionmaking, participants reported that they were able to understand the clearly communicated perspectives of the different decision-making group members. Thus, by practicing CBA, the workshop participants understood the benefits of the structured CBA framework which helps to make better group decisions and additionally helps to understand the decision without being involved in the process. Consequently, CBA should be trained using examples that reflect decisions of the local workshop participants to achieve a high level of pragmatic application with the CBA method. With pre-loading examples from the workshop participants, learning about the CBA method is ensured. Nevertheless, almost all participants struggle in providing the required information for the pre-load, because they do not understand the difference between factor, criterion and attribute without any consistent support. Thus, the follow-up and coaching of the train the trainer concept is a success factor in ensuring quality of CBA facilitation as well as a tangible improvement and confidence in the decision. By attending just one workshop, participants are mostly not able to use CBA correctly without the help of a facilitator. Furthermore, participants struggled in using the vocabulary precisely. Therefore, knowledge providers such as trainers are necessary to support the decision-maker. Only with the intentional strategic broad-scale rollout of decision-making methods will the organizational decision-making culture be inspired and convinced to transform out of their old hierarchical-biased methods and into a more capable and empowered method of collaborative decision-making.

\section{TRANSFER TO THE CONSTRUCTION INDUSTRY}

The construction industry is characterized by a supply chain that is split into several pieces (e.g. Rutten et al. 2009), resulting in knowledge allocation among various regional small and medium-sized companies. The project team therefore also consists of a certain number of different organizations with different cultural backgrounds that must be empowered to make competent decisions that are best for the specific project. The implementation of a new method across the construction industry often needs more time than the implementation across an automotive organization, because more people from different organizations are involved who first need to develop a common understanding. Implementing a method in a company restricts the training area to the number of employees, if the network of suppliers is not considered. In the construction industry, limiting the number to an organization limits the use and the full potential of the method when not applied in projects. However, the construction industry does provide an advantage in spreading the method across the industry, because of its project-based attribute. If project team members experience the benefits, and thus accept and want to apply the method, they will multiply by using the method in future projects. Therefore, when piloting CBA an integrated project team should be trained in and apply CBA for various group decisions in a specific project. Different decisions occur naturally during the 
different project phases and require different members who participate in the decisionmaking process. Thus, during project progress new project participants need to be introduced to CBA. Now, based on the named difference, to what extent is the concept transferable? At the beginning, the expectations and conditions should be aligned in order to create a common mind-set. DT is useful to identify the user's needs and clarify expectations. Therefore, the authors recommend starting with a DT workshop or using parts of the DT workshop to better understand the users and at the same time increase the understanding of why to implement CBA. By doing so, the acceptance will increase. The concept itself could then be transferred 1:1, by considering project-specific topics. Therefore, the content of different elements must be adjusted to the specific project. Examples to train the users should be in the context of the usual decisions they face. Furthermore, attending a workshop will give them insights, but not the confidence to apply CBA correctly. Therefore, at the beginning a facilitator is needed to help the team implement the method correctly and create awareness regarding inclusiveness. Thus, the authors suggest to:

- Start first with the implementation in a specific project, so the benefits of CBA can be communicated within different organizations.

- Start the training with the DT workshop to understand users' needs.

- Adjust the content of the training to the project team or the organizational team.

- Spread the method by transferring experienced team members from project to project.

\section{CONCLUSIONS}

This paper presents an approach of how to implement CBA in project teams as well as across different organizations in the construction industry. Based on the iterative development of an adaptive concept that is partially applied within Daimler AG, using DT and continuous improvement in the meaning of PDCA, it can be concluded that the implementation requires a thoughtful plan that leaves room for adjustments in accordance to the project team's needs. Furthermore, in comparison to the automotive industry, there is another factor that needs to be considered when applying CBA to project teams in the construction industry due to the fact that a construction project involves several participants from different organizations. This increases the complexity and makes it even more necessary to apply a concept that works for the project team. This paper does not give insights into training the trainer or regarding the facilitation of decision-making with CBA itself. Here, more research is needed to support an effective CBA implementation across the construction industry.

\section{REFERENCES}

Arroyo, P., Fuenzalida, C., Alberti, A., and Hallowell, M. (2016). "Collaborating in Decision Making of Sustainable Building Design: An Experimental Study Comparing CBA and WRC Methods." Energy and Building, 128(5), 132-142. 
Arroyo, P., and Long, D. (2018). "Collaborative Design Decisions." In: 26th Annual Conference of the International Group for Lean Construction, Chennai, India.

Baron, J. (2008). Thinking and Deciding. 4th Ed. Cambridge University Press.

Brown, T. (2008). Design thinking. Harvard Business Review, 86(6), 84-92.

Dickens, L., and Watkins, K., (1999). “Action Research: Rethinking Lewin.” Management Learning, 30(2), 127-140.

Elmuti, D., Kathawala, Y., and Wayland, R. (1993). "Traditional Performance Appraisal Systems: The Deming Challenge.” Management Decision, 30(8), 43-48.

Howard, R.A. (1966). Decision Analysis: Applied decision Theory. Proceedings of the Fourth International Conference on Operational Research Wiley-Interscience.

Kelley, T., and Kelley, D. (2013). Creative Confidence - Unleashing the Creative Potential within Us All. Crown Business, New York.

Meinel, C., and Leifer, L. (2011). Design Thinking Research. In: Plattner, H., Meinel, C., and Leifer, L.: Design Thinking. Understand - Improve - Apply. Springer-Verlag, Berlin.

Plattner, H., Meinel, C. and Weinberg, U. (2009). Design Thinking. Innovation lernen, Ideenwelten öffnen. Mi-Wirtschaftsbuch, München, Berlin and Heidelberg.

Rutten, M.E.J., Dorée, A.G., and Halman, J.I.M. (2009). "Innovation and interorganizational cooperation: A synthesis of literature." Constr. Innov., 9(3), 285297.

Schöttle, A., and Arroyo, P. (2017). "Comparison of Weighting-Rating-Calculating, Best Value, and Choosing by Advantages for Bidder Selection." ASCE J. Constr. Eng. Manage., 143(8). https://doi.org/10.1061/(ASCE)CO.1943-7862.0001342.

Schöttle, A., Arroyo, P., and Christensen, R. (2018): "Demonstrating the Value of an Effective Collaborative Decision-Making Process in the Design Phase." In: 26th Annual Conference of the International Group for Lean Construction, Chennai, India.

Suhr, J. (1999). The Choosing by Advantages Decisionmaking System. Quorum, Westport, CT. 